\title{
VALUATION OF CONDITIONS OF MECHANIZED MILKING OF COWS AND OF THE MAMMARY HEALTH SITUATION IN THE EAST CENTRAL DE SOUSSE (TUNISIA)
}

\author{
Y. M'Sadak*, M. Makhlouf, H. Sboui \\ Université de Sousse, Institut Supérieur Agronomique de Chott-Mariem, BP 47- CP 4042, Tunisie.
}

Received: 17 February 2014 / Accepted: 4 August 2014 / Published online: 31 December 2014

\begin{abstract}
The aim of this study is to evaluate mainly the conditions the milking and the mammary health status of cows in the central East Sousse (Tunisian Sahel). The study was conducted on a sample of 20 small and means dairy cattle herds aboveground divided into two study areas. This study examined the general conditions of cattle, the practices of trafficking and the situation mammary health of cows, while completing the analysis by assessing quantitative losses caused in the product milk. The situation detected in the elevages requires an effective response against mastitis while trying to control the risk factors in small and medium farms herds aboveground.
\end{abstract} Keywords: Cattle aboveground; equipment and practices milking; mammary diagnosis; Californian Mastitis Test.

\section{INTRODUCTION}

Les contraintes d'application de la traite mécanique et l'intensification de la production laitière ont favorisé les pathologies mammaires, constituant un problème majeur dans la conduite d'un troupeau de vaches laitières. La maîtrise du taux d'infection mammaire est complexe et passe par la connaissance des différentes épidémiologies et pathogénies des espèces bactériennes mises en cause et par la maîtrise tant de la machine à traire que de l'hygiène de la traite, de l'environnement et de la gestion des traitements des infections [1].

Author Correspondence, e-mail: msadak.youssef@yahoo.fr

Tel.: +21673 327 544; fax: +21673327591.

ICID: 1124382 
L'infection intra-mammaire se traduit, le plus souvent, par une élévation de la concentration en cellules somatiques (CCS). Le diagnostic est basé sur la mesure directe de la CCS ou indirecte (Californian Mastitis Test) des cellules présentes dans le lait. Le Californian Mastitis Test (CMT), encore appelé Test de Schalm, est le test le plus pratique et le plus répandu dans le monde. Il s'agit d'un test semi-quantitatif basé aussi sur la teneur du lait en cellules somatiques [2].

En Tunisie, comme dans la plupart des pays, les mammites bovines constituent une pathologie dominante dans les élevages laitiers. A cet égard, on estime qu'environ $30 \%$ des vaches laitières sont réformées, à cause d'une telle pathologie [3]. Cette situation a été accentuée avec le développement de l'élevage bovin laitier en système hors sol et le recours à la mécanisation de la traite chez les petits éleveurs. Le déroulement correct de la traite permet le maintien de la santé mammaire de la vache et de la production quantitative et qualitative du lait. Tout doit être mis en œuvre pour réaliser une traite calme, complète, rapide et hygiénique.

La présente étude se propose de diagnostiquer la conduite technique et hygiénique de la traite des vaches laitières et d'évaluer la santé des mamelles à partir des Tests périodiques CMT au niveau d'un certain nombre de petites unités laitières menées en hors sol dans la région CentreEst de Sousse, zone littorale semi-aride de la Tunisie.

\section{MATERIEL ET METHODES}

\subsection{Collecte des données}

Le travail s'est intéressé à 20 élevages bovins laitiers totalisant 197 vaches présentes (VP) dont 162 vaches en lactation (VL) dans la région de Sousse, répartis sur deux zones du Centre-Est, à savoir : 15 troupeaux dans la Zone 1 (Kalâa Kébira) et 5 troupeaux dans la Zone 2 (ChottMariem, Sidi Bou Ali et Akouda), durant une période de 3 mois, étalée de mi-février jusqu'à mi-mai 2012.

La traite mécanique biquotidienne en pot est adoptée chez la totalité des exploitations suivies. Toutes les vaches considérées sont de la même race (Frisonne Holstein). Le système d'élevage, du type hors sol, caractéristique de tout le Sahel Tunisien, est déterminé par l'insuffisance, voire l'absence des ressources fourragères, à cause des ressources en eau généralement limitées tant quantitativement (zone semi-aride à pluviométrie généralement $<350 \mathrm{~mm}$ ) que qualitativement (salinité élevée).

Les 20 troupeaux bovins laitiers observés ont une taille de 4 à 22 VP. Ils peuvent être classés comme petits et moyens élevages. 55\% des VP appartiennent à des troupeaux de taille comprise 
entre 4 et 9 , alors que $75 \%$ des VL sont de la même catégorie, ce qui reflète la dominance des troupeaux de petite taille.

Les données de l'étude concernant les conditions d'élevage et de traite ont été recueillies à partir des visites effectuées aux élevages considérés. Il y a eu recours à trois sources d’informations épidémiologiques : les vaches, le milieu d'élevage et de traite ainsi que l'éleveur. Il existe plusieurs moyens d'accès à ces renseignements : Le protocole d'enquête (Conditions d'élevage et de traite), le diagnostic sanitaire mammaire (Comptages cellulaires indirects faisant appel à des Tests CMT), le diagnostic visuel des équipements et des chantiers de traite par l'enquêteur, ... Pour ce faire, un diagnostic épidémiologique des mammites bovines a été effectué sur lait de quartier pour l'évaluation cellulaire indirecte du lait par le CMT (selon trois passages). Auparavant, un suivi des conditions techniques et hygiéniques de la traite mécanique des vaches a été mis en œuvre.

\section{2. Réalisation du CMT}

Le CMT est une méthode rapide, simple et économique pour détecter les infections mammaires au niveau de chaque quartier des mamelles. Il donne indirectement une indication sur la quantité de cellules somatiques présentes dans le lait. La réalisation du CMT a été accomplie selon le protocole présenté par [4] : Il faut tout d'abord s'assurer que les trayons sont exempts de débris et vérifier la présence éventuelle de lait anormal. On adopte toujours la même position pour tenir la palette sous le pis, afin de faciliter le repérage des quartiers lors de l'interprétation et de recueillir du lait de chaque quartier dans le godet correspondant. On incline la palette pour jeter le trop plein et conserver juste assez de lait pour que le niveau atteigne le plus grand cercle concentrique. Puis, on ajoute un volume de $2 \mathrm{ml}$ du réactif (Teepol) équivalent à la quantité de lait en remplissant le godet jusqu'au cercle central et on mélange bien le réactif et le lait par un mouvement circulaire pendant 10-30 secondes. Enfin, on interprète immédiatement le test pour chaque quartier (Tableau 1). 
Tableau 1. Règles d'interprétation des résultats du CMT sur lait de vache par quartier [5]

\begin{tabular}{|c|c|c|c|}
\hline Aspect & Résultat & $\begin{array}{c}\text { Cellules par ml } \\
\mathbf{( x ~ 1 0 0 0 )}\end{array}$ & Interprétation \\
\hline $\begin{array}{c}\text { Aucune réaction : Consistance normale et } \\
\text { couleur grise }\end{array}$ & 0 & $<200$ & Lait normal \\
\hline $\begin{array}{c}\text { Léger gel en flocons disparaissant après } \\
\text { quelques secondes }\end{array}$ & 1 & 200 à 500 & Mammite latente \\
Mammite subclinique \\
Léger gel persistant sous forme de \\
filament grumeleux & 2 & 500 à 1000 & Mammite subclinique \\
Traite irritante
\end{tabular}

\section{RESULTATS ET DISCUSSION}

\subsection{Contexte général de l'étude}

Le système d'élevage bovin laitier dans le Sahel Tunisien est considéré comme étant un système hors sol, en raison des surfaces agricoles réduites destinées à la production fourragère. Les superficies des exploitations visitées varient de 0 à 5 ha dont $30 \%$ des exploitations sans Superficie Agricole Utile (SAU). Les eaux utilisées sont majoritairement propres, soit 90\% (Réseau de Distribution d'eau potable et Sondages), alors que 10\% des eaux (Citernes) sont sales, à cause de stagnation ou de nettoyage non fréquent des bassins d'eau.

Il y a une grande variabilité entre les éleveurs au niveau des rations présentées aux vaches. Comme aliment grossier, on trouve la paille (10\%), le foin (90\%). L'herbe ou le fourrage vert sont distribués dans $65 \%$ des cas. Pour le concentré, on trouve le concentré $\mathrm{N}^{0} 7$ des vaches laitières (90\% utilisé par tous les élevages), le concentré №5 d'engraissement (10\%) utilisé aussi dans les rations des vaches laitières pour avoir des veaux de bonne qualité bouchère et aussi vu son prix moins élevé ainsi que le son ( $80 \%$ des élevages) et les bouchons de luzerne ( $5 \%$ des élevages). 


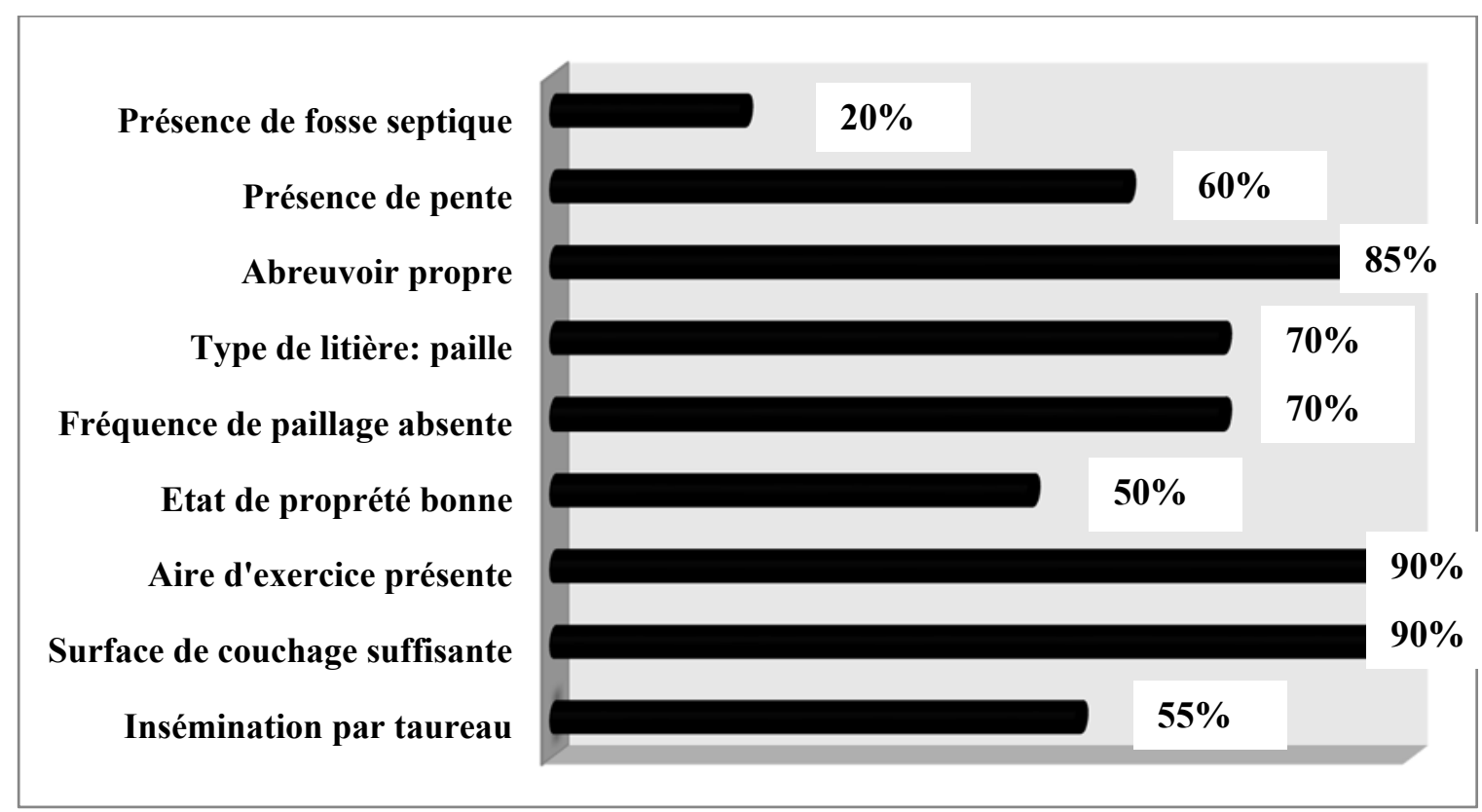

Fig.1. Quelques conditions relevées d'élevage bovin laitier

L'insémination est appliquée selon les deux méthodes courantes, à raison de $45 \%$ pour l'insémination artificielle, contre 55\% pour l'insémination naturelle (Figure 1). Tous les élevages enquêtés sont à stabulation libre. Les bâtiments sont généralement répartis en des aires d'exercice non couvertes et non paillées, des aires de couchage aussi non couvertes et non paillées, des aires d'alimentation couvertes et souvent utilisées en même temps pour la traite, vu que les aliments sont distribués généralement juste avant la traite. La figure 1 montre aussi certaines conditions d'élevage rencontrées, où l'on remarque $90 \%$ des exploitations ayant une aire d'exercice et une surface de couchage suffisantes, ce qui assure un environnement relativement confortable, avec tendance vers le respect du bienêtre animal nécessaire à la survie des vaches et à la sécrétion lactée suffisante.

\section{2. Évaluation des équipements et pratiques de traite des vaches}

\subsubsection{Machines à traire adoptées}

De par sa capacité à véhiculer les microbes, la machine à traire peut avoir un rôle infectant lié à des retours de lait contaminé vers le canal du trayon. C'est le cas lors des phénomènes d'impact, de traite humide et de surtraite qui provoquent ou facilitent la pénétration des microorganismes dans le canal du trayon. Le fonctionnement de la traite mécanique est basé sur l'équilibre parfait d'un trinôme : le trayeur, la vache et la machine à traire. Le rôle des partenaires est clair : au trayeur de s'assurer de l'hygiène des trayons et de provoquer la descente du lait ; à la machine à traire ensuite de récolter ce lait de manière hygiénique et non agressive pour les trayons, de l'acheminer et de le stocker sans en altérer la qualité [6], à la vache de 
collaborer pour assurer l'harmonie recherchée [7]. Pour assurer ceci, il faut contrôler, entre autres, l'efficacité, la propreté et le bon fonctionnement des machines adoptées.

Les 22 machines adoptées (utilisées dans 20 exploitations bovines laitières étudiées) sont du type système de Traite Mécanique en Pot (TMP). Toutes les installations de traite sont du type chariot-trayeur (installation mobile). La marque Tecnosac, d'origine Italienne, est la plus répandue avec un taux de 35\%. En se basant sur l'étude accomplie dans la région de Boumerdes par M'Sadak et al [8], on peut dire que la marque Tecnosac est la plus adoptée dans cette région (39\%). De même, dans la région de Kalâa Kébira, M'Sadak et al [9] ont révélé que Tecnosac est la marque la plus utilisée (40\%). Il ressort donc que dans les régions du Sahel Tunisien, cette marque italienne est privilégiée par les éleveurs.

La plus récente machine date depuis un an, alors que la plus ancienne date de 15 ans (20\%). En avançant en âge, le risque de la machine à traire sur la mamelle augmente, donc, il faut en permanence l'entretenir et la contrôler au moins une fois par an par un Agent spécialisé [7].

En outre , 90\% des machines à traire ont un Faisceau-Trayeur (FT) et un Pot Trayeur (PT). Ceci peut être lié entre autres à la production moyenne des vaches en lactation qui ne dépasse pas 22 1/vache/jour, à la taille relativement faible des troupeaux laitiers suivis.

\subsubsection{Chantiers mécanisés de traite}

\subsubsection{Diagnostic du déroulement des opérations de traite}

La technique de traite est un point clef dans la transmission des germes dits de réservoir mammaire : staphylocoques et streptocoques pour l'essentiel. Elle est également importante pour prévenir les infections environnementales. La mise en place d'une technique rigoureuse de traite est essentielle pour réduire le risque de mammite [10]. A cet égard, des suivis techniques et hygiéniques des chantiers de traite ont été réalisés chez les 20 élevages bovins laitiers hors sol.

Les 20 exploitants visités nettoient la mamelle en utilisant l'eau (75\%) et une serviette collective. Sous prétexte d'améliorer la qualité de nettoyage, les éleveurs ajoutent des petits morceaux de savon "vert" dans l'eau de nettoyage (10\%), mais, cette amélioration reste insuffisante, compte tenu de l'utilisation des lavettes collectives. L'essuyage se fait aussi par la même serviette. De telles opérations ont pour but, entre autres, d'éliminer les microbes liés à la mamelle, de stimuler la sécrétion lactée, et de profiter au maximum de l'effet de l'ocytocine. 95\% des éleveurs éliminent les premiers jets sur le sol sous la vache, présentant ainsi un facteur de risque de contamination de la surface de couchage. Les premiers jets de lait portent une 
charge microbienne importante qui peut constituer un des principaux polluants du lait de mélange qu'il faut éliminer [4].

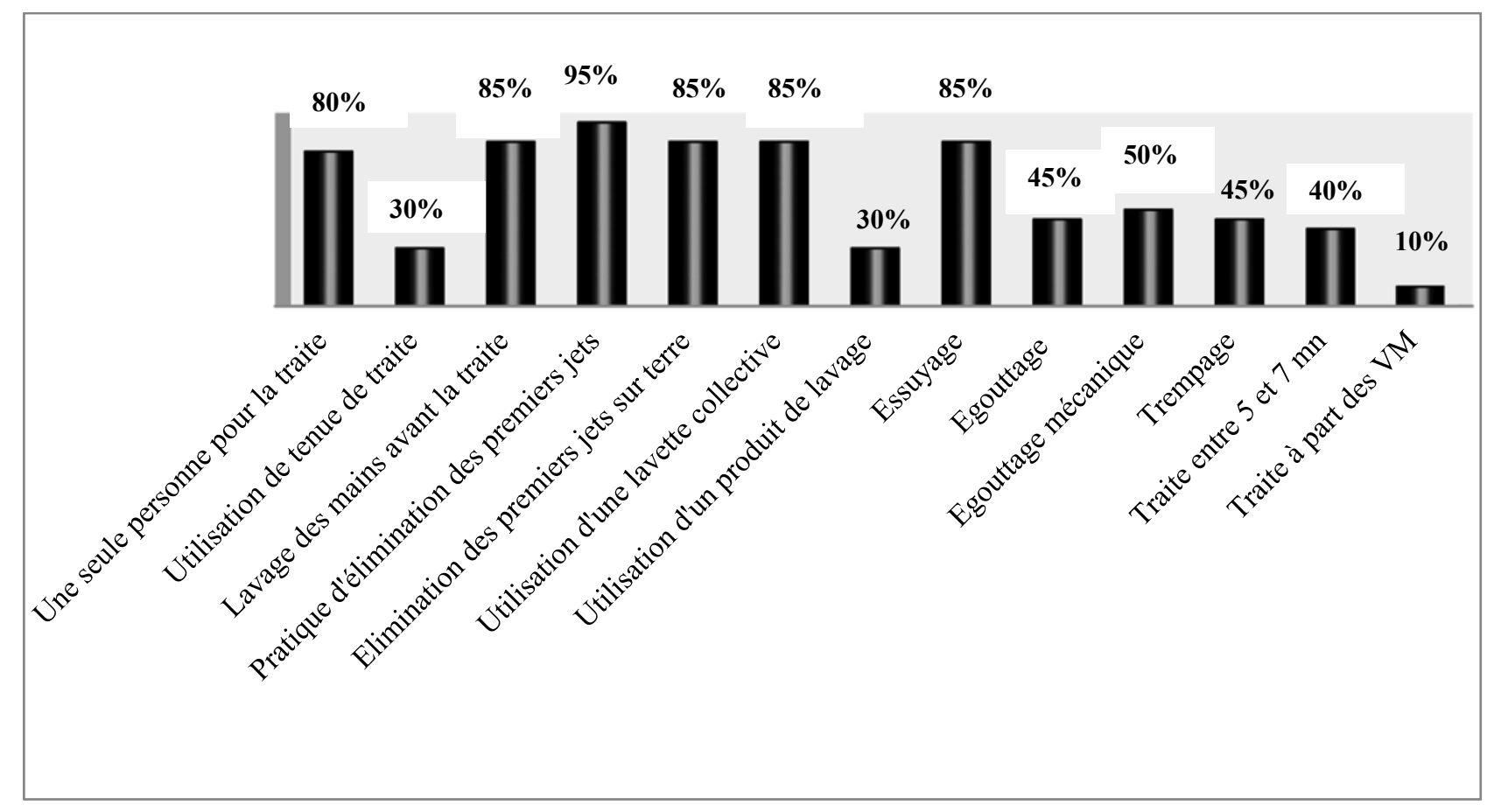

VM : Vaches Mammiteuses

Fig.2. Diagnostics technique et hygiénique des chantiers mécanisés de traite des vaches

L'égouttage est une pratique qui, à la fin de la traite, consiste à extraire le lait que la machine n'a pas pu évacuer [11]. L'égouttage est pratiqué chez $45 \%$ des élevages considérés. L'égouttage est mécanique chez $50 \%$ des élevages engagés.

Après la traite, il est fortement recommandé de désinfecter les trayons [12]. Seulement $45 \%$ des éleveurs assurent la désinfection des trayons après la traite (Figure 2). Cette désinfection est faite uniquement par trempage (pulvérisation non appliquée) dans une même solution antiseptique, sans tenir compte que le choix du produit de trempage se fait en fonction des paramètres spécifiques à chaque élevage, comme le type de mammites rencontrées, l'état des trayons, ... 100\% de ces désinfections sont régulières, après chaque traite, l'éleveur fait tremper les trayons dans un produit à base d'iode. L'étude accomplie par Enault [6] a révélé qu'il existe une relation entre l'apparition des mammites et le mauvais état des trayons non désinfectés. Néanmoins, le trempage contribue à refermer l'orifice à l'extrémité des trayons après la traite, en plus de l'inactivation des bactéries pour empêcher leur pénétration dans le canal du trayon. Il a été démontré que le trempage des trayons dans un produit efficace, peut prévenir jusqu'à $50 \%$ des nouvelles mammites [13]. 


\subsubsection{Conduite de la traite}

Pour apprécier la qualité de la conduite de la traite, on doit observer notamment l'hygiène, la rapidité, la tranquillité et l'égouttage. On dit qu'une :

- Traite est hygiénique, quand le trayeur respecte l'ordre de traite ainsi que la propreté du lieu de traite, de la tenue de traite et du matériel de traite.

- Traite est rapide, si le temps de traite ne dépasse pas $6 \mathrm{mn}$ par vache et $1 \mathrm{~h} 30 \mathrm{mn}$ par troupeau.

- Traite est calme, lorsque la traite est réalisée sans coups, ni bruits et ni chocs électriques.

- Traite est complète, quand l'égouttage de la mamelle est exécuté sans dépasser les $30 \mathrm{~s}$ pour éviter le phénomène de surtraite.

- Surtraite est un phénomène qui résulte d'un défaut de vigilance de la part de l'éleveur ou d'une traite plus aisée d'un quartier par rapport aux autres qui sera donc vidé auparavant. Ceci augmente le risque de lésions du trayon et le reflux de lait dans la mamelle [10].

La figure 3 révèle que $95 \%$ des éleveurs font une traite relativement hygiénique et tous appliquent une traite calme. Par contre, la traite est considérée comme rapide et complète respectivement dans $70 \%$ et $75 \%$ des chantiers mécanisés. La surtraite a été observée dans $20 \%$ des cas.

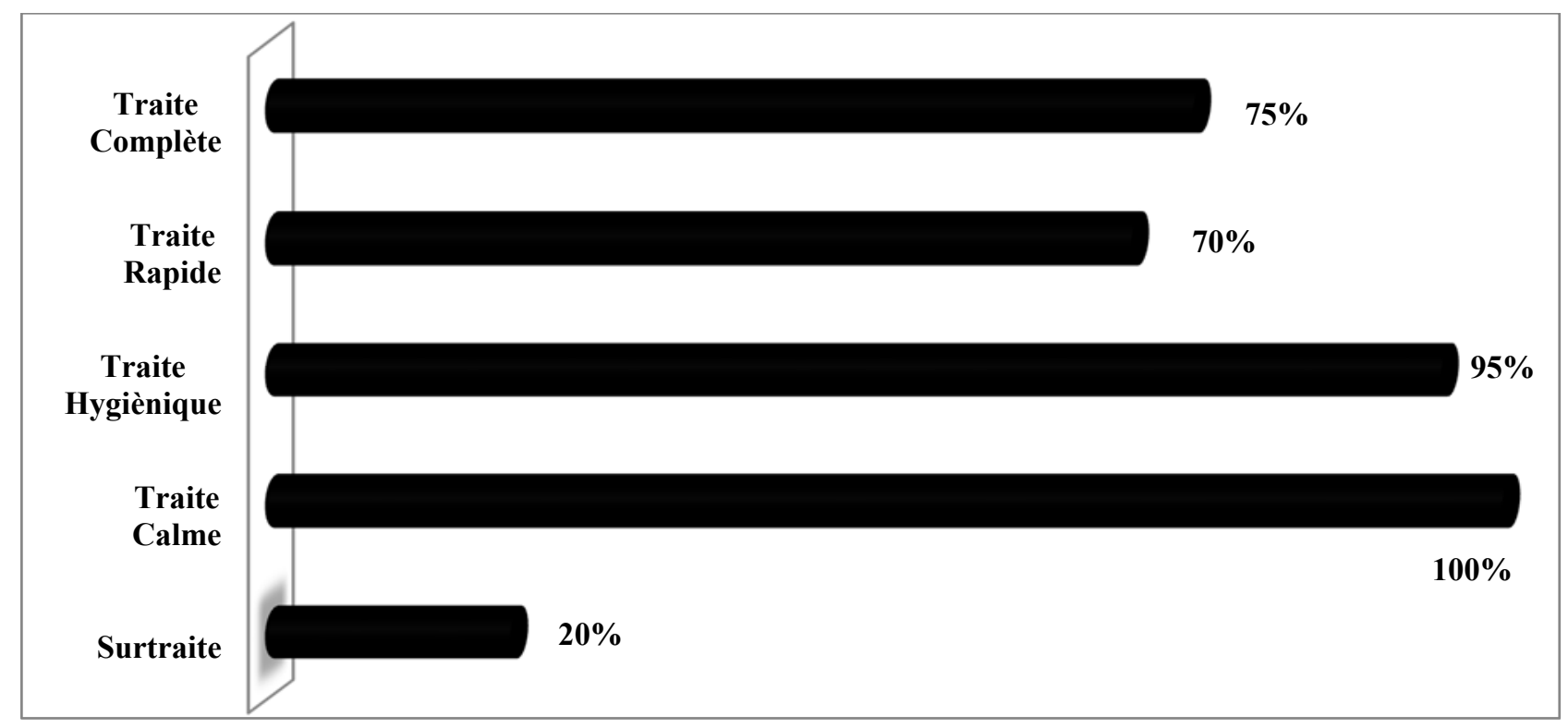

Fig.3. Diagnostic de la conduite technique et hygiénique de la traite des vaches

\subsubsection{Nettoyage des équipements de traite adoptés}

Le nettoyage de la machine à traire est un élément clé pour la production de lait de bonne qualité. Une machine à traire mal entretenue peut augmenter la fréquence de nouvelles infections mammaires soit par un rôle vecteur de germes pathogènes depuis les quartiers 
infectés vers les quartiers sains, soit par son rôle traumatisant sur le canal du trayon (mauvais état des manchons-trayeurs), en diminuant son effet barrière [4].

Les éleveurs visités adoptaient le nettoyage de leurs machines selon différentes actions. Le nettoyage des machines à traire à l'aide d'un détergent avec alternance acide/alcalin est pratiqué par 5\% des éleveurs alors que le nettoyage de 10\% des machines est réalisée à l'aide d'un détergent sans alternance acide/base. Ce résultat est relativement loin de celui trouvé par M'Sadak et al [9] lors de l'étude effectuée dans la région de Kalâa Kébira, où ils ont relevé que $15 \%$ des machines sont nettoyées par l'eau additionnée d'un détergent avec alternance acide/alcalin. Une étude accomplie par Billon et al [14] a dévoilé qu'un simple rinçage à l'eau froide pendant $3 \mathrm{mn}$ n'entraînait pas de diminution sensible du nombre des bactéries présentes dans les faisceaux-trayeurs, alors qu'une circulation d'eau à $74{ }^{\circ} \mathrm{C}$ pendant $3 \mathrm{mn}$ ou à $85^{\circ} \mathrm{C}$ pendant $5 \mathrm{~s}$ à travers les faisceaux- trayeurs réduisait la charge bactérienne de 97 à 100\%. À ce propos, la température de la solution de lavage est l'un des facteurs de réussite du nettoyage qui devrait être strictement respecté, sous peine d'une perte d'efficacité. Dans la quasi-totalité des cas, le cycle de nettoyage de la machine à traire était loin d'être appliqué convenablement, d'autant plus que les facteurs de succès d'un bon nettoyage ne sont pas tout à fait révérés (qualité et température de l'eau de lavage, dose de détergent, temps de contact). Toutefois, il convient de signaler que $85 \%$ des éleveurs appliquaient une action mécanique lors du nettoyage, qui consistait à faire agiter le faisceau-trayeur dans un seau contenant de l'eau mélangée avec la solution détergente ou avec la solution désinfectante (eau + javel), et ainsi, la tuyauterie est nettoyée par aspiration de ce mélange, après la mise en marche de la machine à traire. Par ailleurs, il convient aussi de noter que les éleveurs pratiquent les opérations de nettoyage du matériel : lavages juste avant la traite et après la traite respectivement dans $25 \%$ et $85 \%$ des cas (Figure 4).

L'étude menée dans la région de Mahdia par M'Sadak et al [15] a montré que les résultats relevés au niveau des diagnostics hygiénique et technique des chantiers de traite mis en œuvre semblent indiquer que les conditions d'hygiène de la traite sont loin d'être respectées dans l'ensemble avec environ la moitié des éleveurs pratiquant une traite pouvant être considérée plus ou moins hygiénique. Les mauvaises conditions hygiéniques de traite (lavette collective, sans essuyage des trayons, sans élimination des premiers jets de lait avant la traite, sans désinfection des trayons après la traite, nettoyage incorrect du matériel de traite) pourraient constituer les probables facteurs essentiels de risque des mammites avec également le non respect des paramètres de montage et de fonctionnement des machines à traire adoptées. 
Respect de l'action mécanique de lavage

Respect de dose préconisée de détergent

Respect de température de l'eau de rinçage

Post-rinçage avec de l'eau

Rinçage de la machine avec eau + javel

Pré-rinçage après chaque traite

Rinçage de la machine avant chaque traite

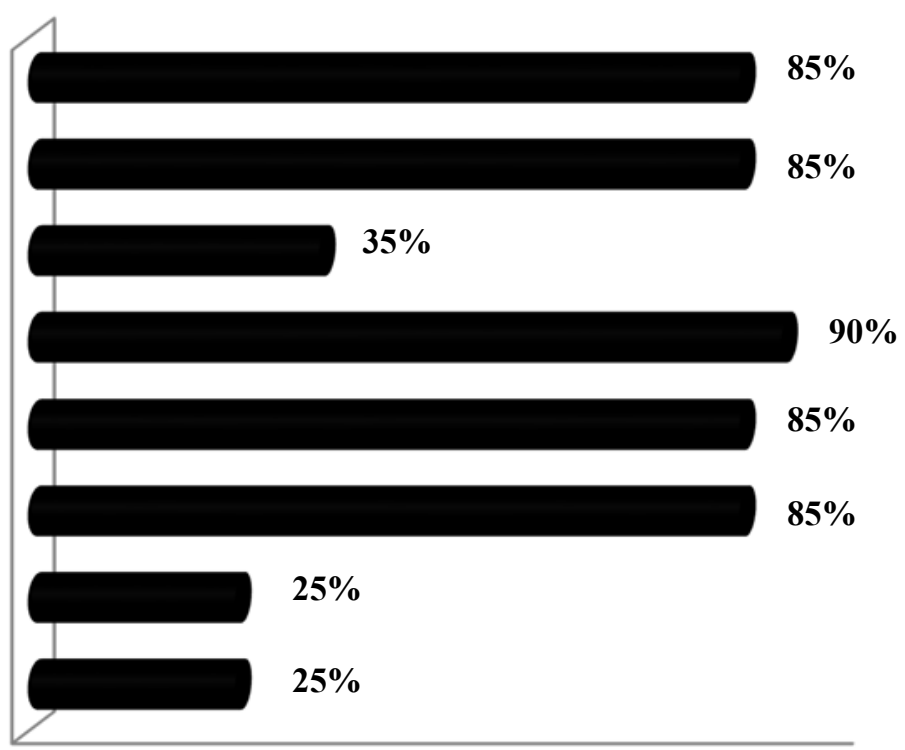

Fig.4 . Diagnostics technique et hygiénique du nettoyage des machines à traire

\section{Appréciation de l'état sanitaire mammaire par le CMT}

\subsection{Selon le passage}

Sur les 623 quartiers contrôlés des vaches durant les trois passages, on a 268 quartiers sains (test négatif), 348 quartiers infectés et 7 quartiers non fonctionnels (NF). On remarque qu'environ 56\% des quartiers fonctionnels (616) sont infectés (Figure 5). Ce niveau d'infection est proche de celui trouvé dans la région de Monastir par Ben Omrane [16], où l'on a trouvé un taux d'infection atteignant $60 \%$, mais considéré comme grave par rapport à celui relevé dans toute la région de Sousse par Hamed [17] ne dépassant pas $40 \%$ pendant uniquement deux contrôles. Signalons que cette comparaison concerne des élevages du Sahel Tunisien conduits dans des conditions similaires. A cet égard, en se basant sur l'étude effectuée par M'Sadak et al [18], le taux d'infection mammaire relevé dans la région de Mahdia s'est révélé le meilleur $(37 \%)$.

Par ailleurs, la situation sanitaire mammaire étudiée parait assez plus prononcée que celle rapportée par Saidi et al [19], sur un échantillon de 15 petits élevages de 3 à 25 vaches par exploitation appartenant au Centre de l'Algérie, où le taux des vaches présentant un CMT positif a dépassé $50 \%$. La prévalence de la mammite observée dans une autre étude, réalisée aussi en Algérie par Saidi et al [20], était largement moindre (environ 30\%) dans un contexte différent de celui de la précédente étude. La différence sanitaire mammaire relevée pourrait découler, entre autres, de la race (locale, améliorée et importée en Algérie, contre exclusivement 
importée en Tunisie) et du système de traite adopté (quasi-manuel en Algérie, contre majoritairement mécanique en Tunisie).

On peut dire que l'état sanitaire des mamelles des vaches suivies dans la région d'étude est préoccupant et mérite plus d'assistance et d'encadrement auprès des éleveurs.

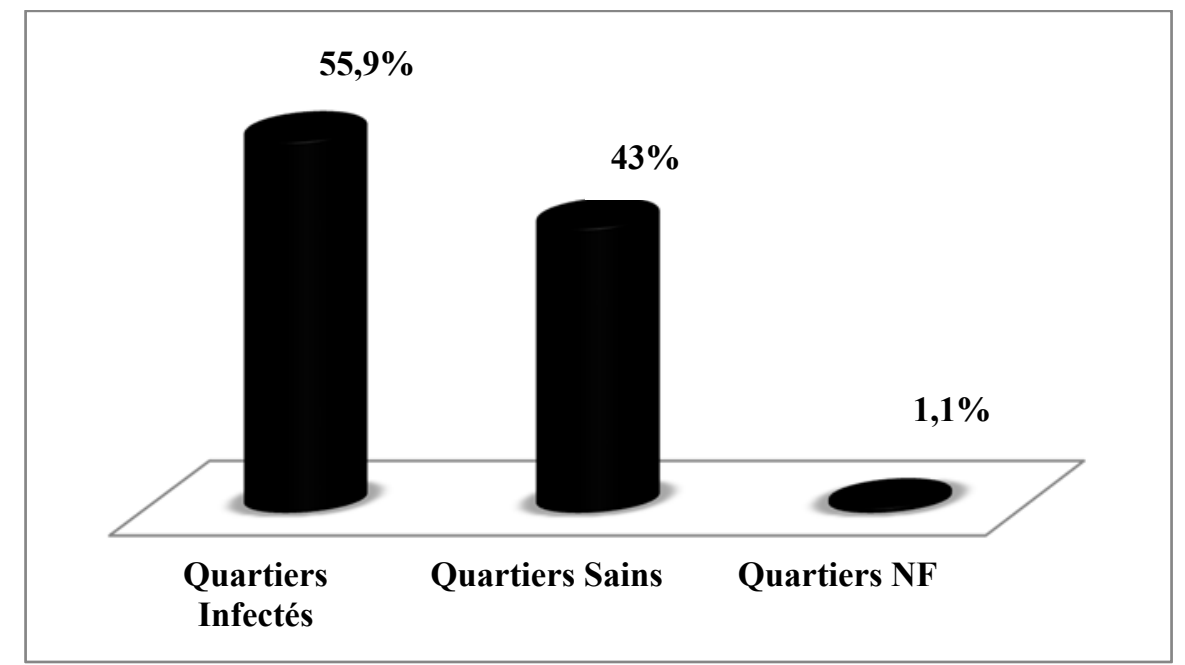

Fig.5. Répartition des quartiers selon leur état d'infection

Il convient aussi de noter une évolution des résultats CMT selon chaque passage (Figure 6). En effet, le taux d'infection a augmenté entre les trois passages réalisés pour tous les types des mammites (scores de 1 à 4), ce qui pourrait être expliqué par le fait de l'élévation de la température ambiante qui a favorisé la diminution relative du nombre des vaches saines et le taux d'infection latente (score1), l'élévation remarquable surtout du taux d'infection subclinique bénigne (score 2) et l'infection subclinique grave (score 3 ).

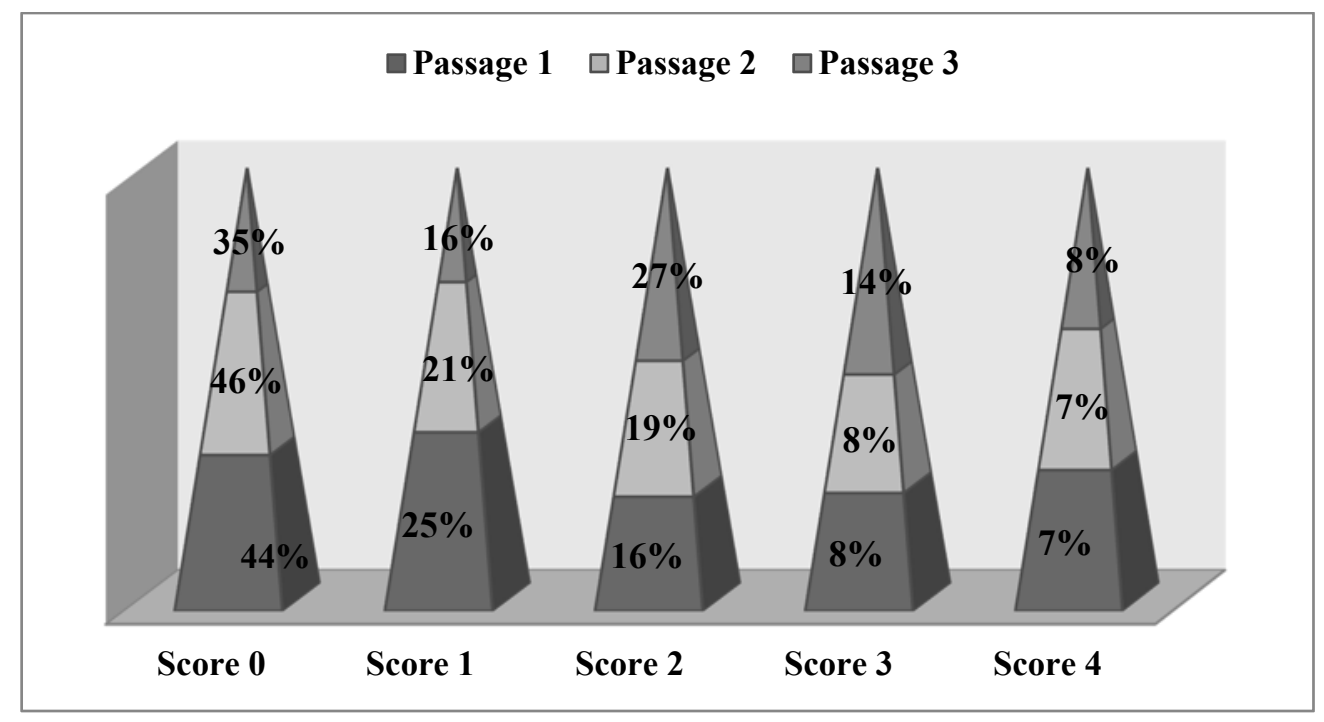

Fig.6. Distribution des scores CMT entre les passages 


\subsection{Selon le quartier}

Les quartiers postérieurs sont plus exposés aux infections mammaires subcliniques latentes (Figure 7). Ces infections sont aussi nombreuses pour le score 1 (52\%), que pour le score 3 relatif aux mammites subcliniques graves (55\%). Il en est de même pour les infections mammaires cliniques relatives au score 4 (62\%). Porcher [21] a révélé que les quartiers postérieurs sont plus souvent infectés que les quartiers antérieurs, ceci est expliqué par le fait que les quartiers postérieurs stagnent plus que les quartiers antérieurs dans les excréments, et aussi à ce que, au repos, ils se trouvent comprimés davantage que les quartiers antérieurs entre les cuisses de la vache. Par contre, Kemp et al [22] ont annoncé que le risque d'infection des quartiers est le même pour les positions postérieures et antérieures, ce qui infirme les résultats CMT trouvés dans le contexte considéré.

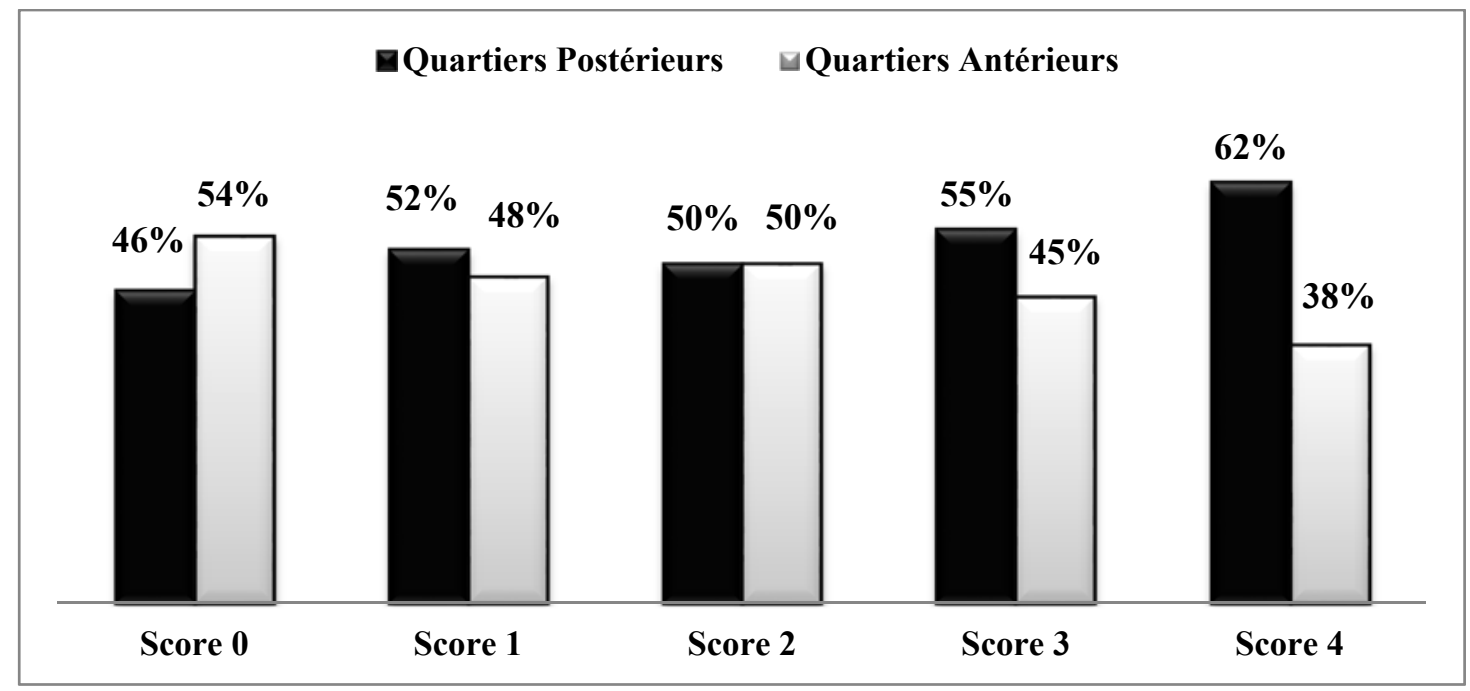

Fig.7. Partage des infections mammaires selon le score et les quartiers antérieurs et postérieurs

Pour les positions droites et gauches (Figure 8), nous avons remarqué que les infections mammaires subcliniques graves (55\%) et subcliniques latentes (51\%) sont observées au niveau de la position gauche. Porcher [21] a affirmé que la situation mammaire serait due à ce que la plupart des bovins choisissent plutôt le côté gauche que le côté droit pour se reposer. L'animal évite de se coucher sur le côté droit, afin de ne pas comprimer le reste des organes digestifs avec la panse, dont la masse est considérable. Cette considération n'est pas tout à fait vérifiée pour les autres scores. 


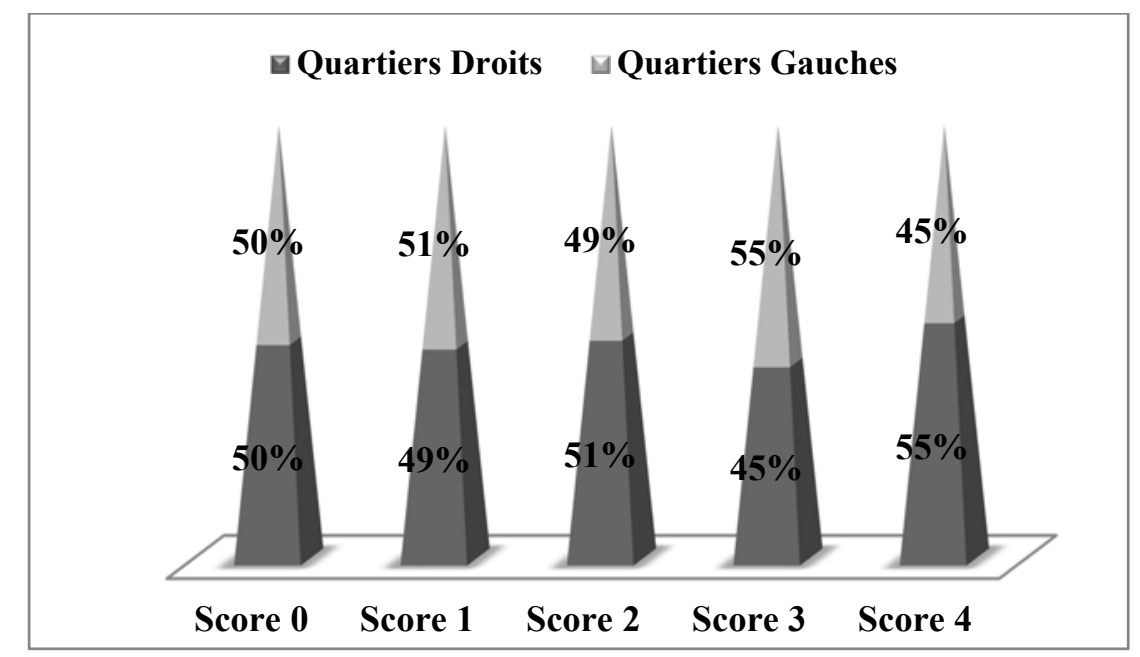

Fig.8. Répartition des infections mammaires selon le score et les quartiers gauches et droits

\subsection{Selon la taille du troupeau}

En outre, nous avons remarqué que le taux d'infection était plus élevé dans les petits troupeaux $(<14 \mathrm{VL})$ avec un pourcentage des vaches présentant les scores CMT de 1 à 4 de 33,2\% (5 à $9 \mathrm{VL}$ ) et 45,7\% (10 à $14 \mathrm{VL})$, contre 32\% dans les autres élevages (>14 VL) (Figure 9). Ceci pourrait être expliqué par la bonne gestion des troupeaux de taille supérieure à $14 \mathrm{VL}$ et la facilité de transmission des infections mammaires dans les petits troupeaux techniquement moins assistés.

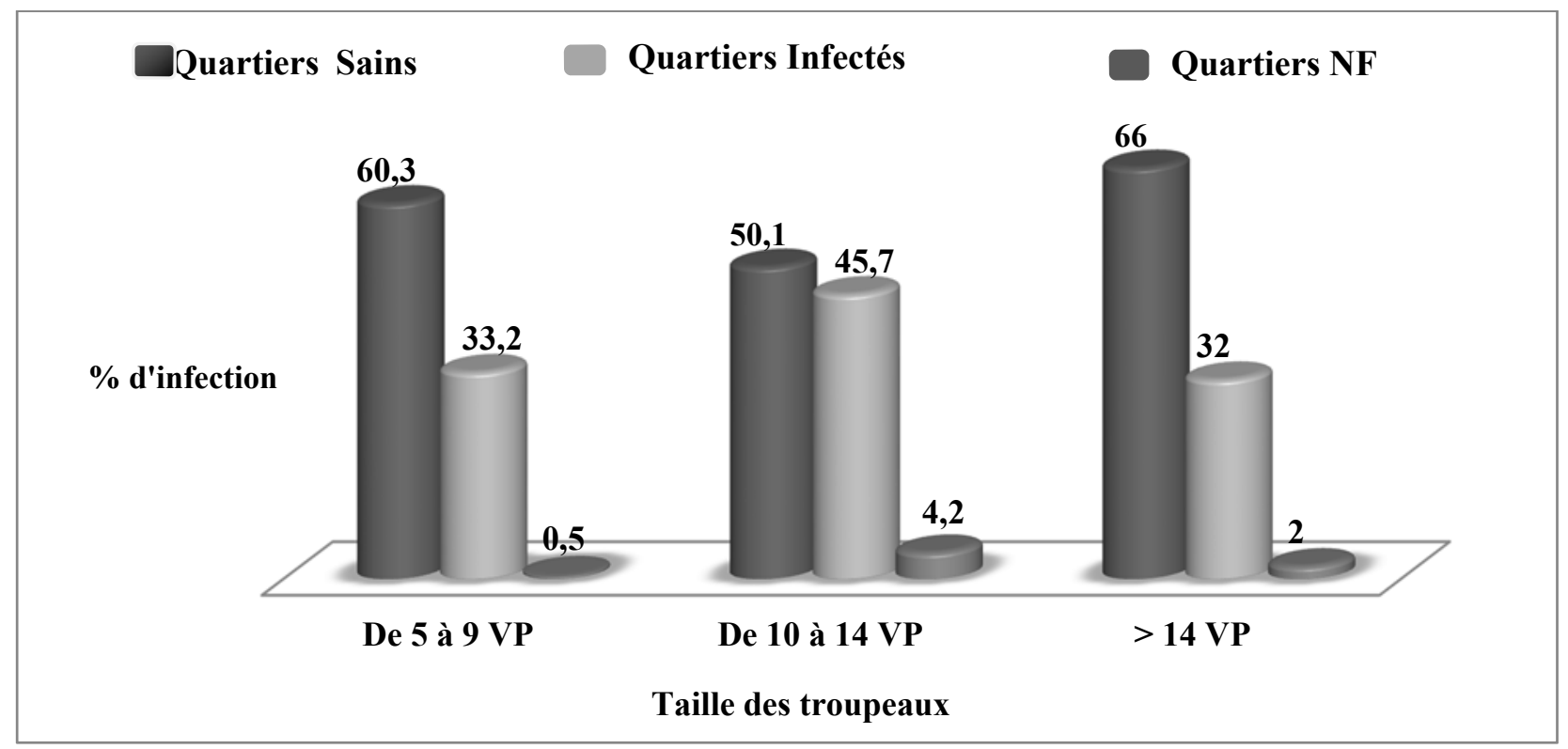

Fig.9. Variation de l'état sanitaire mammaire des vaches selon la taille des troupeaux 


\section{CONCLUSION}

Il ressort de cette étude, réalisée sur un échantillon d'une vingtaine des petits et moyens troupeaux bovins dans la région du Centre-Est de Sousse (Sahel Tunisien), que les conditions de traite s'éloignent des bonnes pratiques chez plusieurs éleveurs (70\% des trayeurs sans tenue de traite, utilisation d'une lavette collective et non individuelle chez $85 \%$ des éleveurs, non désinfection des trayons après la traite chez $55 \%, \ldots$ ) et elles méritent davantage de diagnostic et d'assistance technique. La plupart des éleveurs adoptent une traite hygiénique, rapide et complète, alors que la totalité applique une traite calme. Cependant, le nettoyage du matériel de traite est loin d'être maîtrisé, étant donné que le cycle préconisé n'est que partiellement respecté. Les résultats relevés au niveau du diagnostic sanitaire mammaire par le CMT montrent que $56 \%$ des quartiers testés sont infectés. Le CMT reste le test préférable chez les vaches laitières pour déceler les mammites subcliniques [23]. Il donne une réponse qualitative sur le statut de chaque quartier de la mamelle (saine ou infectée) et permet de sélectionner les animaux sur lesquels seront opérés des prélèvements lors d'enquêtes sur les mammites [24]. Il a l'avantage d'être peu coûteux, de pouvoir être accompli par l'éleveur et de fournir une réponse immédiate. En effet, le CMT constitue une méthode de choix pour les éleveurs et les vétérinaires pour déterminer le statut des vaches vis-à-vis des mammites [25] avant de spécifier un traitement. L'effet de la mammite sur la production laitière dépend notamment de la qualité de sa détection et de celle des thérapies utilisées, ainsi que de la politique de tarissement et de réforme de l'éleveur [26].

Finalement, la mise en place d'un plan de maîtrise des mammites bovines à l'échelle nationale devient de plus en plus une nécessité pour réduire autant que possible la concentration en cellules somatiques du lait au sein du troupeau. Dans l'immédiat, il convient, d'une part, d'améliorer davantage l'hygiène et la technique de traite, le nettoyage et l'entretien des machines à traire, le réglage des paramètres de fonctionnement et l'assistance et l'encadrement des éleveurs, et d'autre part, de garantir le testage complet de fonctionnement au moins annuellement et le service maintenance (réparations, ....).

\section{REFERENCES BIBLIOGRAPHIQUES}

[1] Dumas E., Activité dermatologique de deux nouveaux produits de trempage du trayon chez la vache laitière, Doctorat Vétérinaire, ENV Lyon, France, 2004, 134 p. http://www2.vetagro-sup.fr/bib/fondoc/th_sout/dl.php?file=2004lyon081.pdf 
[2] Bouaziz O., Contribution à l'étude des infections intramammaires de la vache laitière dans l'Est Algérien, Doctorat d'Etat, Université Mentouri Constantine, Algérie, 2005, 296 p. www.umc.edu.dz/theses/veterinaire/BOU4260.pdf

[3] Bachta MS., Laajimi A., Adéquation de l'offre et de la demande des produits laitiers en Tunisie : une analyse micro-économique. In Symposium International sur les filières lait en Méditerranée : enjeux pour un futur durable. Hammamet, 26-28 octobre 2000. Tunisie. Eu. Assoc. Anim. Prod., 2003, 99, p. 392-400.

[4] Boudry B., Traire un lait de qualité : Une attention de tous les jours - Qualité du lait et gestion du troupeau. DGA - Direction du Développement et de la Vulgarisation, 2005, 13 p. http://agriculture.wallonie.be/apps/spip_wolwin/IMG/pdf/Boudry-henri-chap05.pdf

[5] Berthelot X., Lebret P., Petit C, Les infections mammaires de la vache laitière. ENV Toulouse, France, 1987, 192 p.

[6] Enault C., La machine à traire : Recherches et innovations depuis les années 1980 en vue d'améliorer la qualité du lait et la santé de la mamelle chez les vaches laitières. Revue bibliographique, Doctorat Vétérinaire, ENV Alfort, France, 2008, 217 p.

http://theses.vet-alfort.fr/telecharger.php?id=869

[7] M’Sadak Y., Technologie de la Traite des Petits Troupeaux Bovins Laitiers. Session de Formation (Contrôleurs et Réparateurs des Machines à Traire). Centrale Laitière Mahdia, Tunisie, Mars 2009, 75 p.

[8] M'Sadak Y., Krichi A., Kraiem K., Diagnostics Technologique, Technique et Hygiénique de la Traite Mécanique en Pot dans la Délégation Boumerdes, Poster, Actes $16^{\text {ème }}$ Journées Scientifiques IRESA, Nabeul, Tunisie, 2 et 3 Décembre 2009.

[9] M'Sadak Y., Haj Mbarek R., Kraiem K., Diagnostics Technologique, Technique et Hygiénique de la Traite Mécanique en Pot dans la Délégation Kalâa Kébira, Poster, Actes $16^{\text {ème }}$ Journées Scientifiques IRESA, Nabeul, Tunisie, 2 et 3 Décembre 2009.

[10] Mezine MCS., Analyse descriptive des facteurs de risque lies aux mammites dans des élevages d'une clientèle des Ardennes appliquant la démarche GTV partenaire , Doctorat Vétérinaire, ENV Alfort, France, 2006, 95 p. http://theses.vet-alfort.fr/telecharger.php?id=119 http://www.gov.on.ca/OMAFRA/french/engineer/facts/97-190.htm

[11] Hollis B.W., Draper H.H., Burton J.H., Etchers R.J., A hormonal assessement of bovine parturient paresis: evidence for a role oestrogen, J. Endocrinol., 1981, 88: 161-171.

[12] Baudry Ch., Mercier P., Mallereau M-P., Lenfant D., Évaluation de l'efficacité du posttrempage chez la chèvre, Rev. Med. Vet. 2000, 151, p. 1035-1040. 
[13] Garland G.A., Technique de traite normale. Fiche technique du Ministère de l'Agriculture et de l'Alimentation de l'Ontario, Canada, 1997, Agdex 410/725.

[14] Billon P et Chapelle H., L'état des trayons : Révélateur de la routine de traite et du fonctionnement de la machine à traire. Journée Qualité de la Traite, Belgique, 2005, 25 p. http://agriculture.wallonie.be/apps/spip wolwin/IMG/pdf/L Etat des trayons revelateur.pdf [15] M'Sadak Y., Mighri L., Kraiem K., Étude de la situation sanitaire mammaire à partir des taux cellulaires de troupeau et estimation des pertes laitières engendrées chez des ateliers bovins hors sol en Tunisie. Revue « Nature et Technologie », N 04/Janvier 2011, p.8-14.

http://www.univ-chlef.dz/RevueNatec/art_04_01.pdf

[16] Ben Omrane H., Évaluation des Conditions de Traite des Vaches, de la Situation Sanitaire Mammaire et des Pertes Quantitatives en Lait dans le Gouvernorat de Monastir. Projet de Fin d'Études. Institut Supérieur Agronomique de Chott-Mariem, Tunisie, 2010, 98 p.

[17] Hamed I., Évaluations Sanitaire, Technique, Technologique et Hygiénique des Conditions de Traite Mécanique en Pot des Vaches dans la Région de Sousse. Projet Fin d’Études. Institut Supérieur Agronomique de Chott-Mariem, Tunisie, 2011, 71 p.

[18] M'Sadak, Y., Mighri L., Kraiem K., Diagnostic de la santé mammaire des vaches laitières chez les petits éleveurs dans la région de Mahdia. Poster- Actes $15^{\text {ème }}$ Journées Scientifiques IRESA, Tunisie, 18 et Décembre 2008.

[19] Saidi R., Khelef D., Kaidi R, Évaluation d'un test de dépistage précoce des mammites cliniques des vaches, Revue d'élevage et de médecine vétérinaire des pays tropicaux, 63 (3-4), 2010, p. 57-61. http://remvt.cirad.fr/cd/derniers num/2010/REMVT10 057 061.pdf

[20] Saidi R., Khelef D., Kaidi R., Bovine mastitis: Prevalence of bacterial pathogens and evaluation of early screening test, African Journal of Microbiology Research, 26 February, 2013, Vol. 7(9), 777-782.

http://academicjournals.org/article/article1380638672_Saidi\%20et\%20al.pdf

[21] Porcher Ch., L'infection latente de la mamelle et ses réveils. Les moyens de la dépister, Revue Le Lait, Tome XII, N¹14, 1932, p. 257-269.

[22] Kemp MH, Nolan AM, Cripps PJ, Fitzpatric JL, Animal based measurements of the severity of mastitis in dairy cows - Veterinary Record, 2008, 163: 175-179.

http://connection.ebscohost.com/c/articles/34107471/animal-based-measurements-severitymastitis-dairy-cows

[23] Ruegg P.L., Reiman D.J., Milk quality and mastitis tests, Bovine Practitioner, 2002, 36: 41-54. 
[24] Gonzales R.N., Jasper D.E., Farver T.B., Bushnell R.B., Franti C.E., Prevalence of udder infections and mastitis in 50 California dairy herds. J. Am. med. Assoc., 1988, 193: 323-328. [25] Busato A., Trachsel P., Schallibaum M., Blum J.W., Udder health and risk factors for subclinical mastitis in organic dairy farms in Switzerland. Prev. Vet. Med., 2000, 44: 205-220. [26] Bartlett P.C., Van Wijk J., Wilson D-J., Green C.D., Miller G.Y., Majewski G.A., Heider L.E. J. Dairy Sci, . 1991, 74: 1561-1572.

\section{How to cite this article:}

M'Sadak Y. Makhlouf M. Sboui H. Valuation of conditions of mechanized milking of cows and of the mammary health situation in the east central de Sousse (Tunisia). J Fundam Appl Sci. 2014, 6(2), 127-143. 